\title{
Closed-loop next generation laser polishing
}

Rolf Rascher, Christian Vogt, Oliver Fähnle, DaeWook Kim

Rolf Rascher, Christian Vogt, Oliver Fähnle, DaeWook Kim, "Closed-loop next generation laser polishing ," Proc. SPIE 10829, Fifth European Seminar on Precision Optics Manufacturing, 108290M (7 August 2018); doi:

$10.1117 / 12.2318749$

SPIE Event: Fifth European Seminar on Precision Optics Manufacturing, 2018, Teisnach, Germany 


\title{
Closed-loop next generation laser polishing
}

$\underline{\text { Rolf Rascher }^{1}}$, Christian Vogt ${ }^{1}$, Oliver Fähnle ${ }^{2}$ and DaeWook Kim ${ }^{3}$

\author{
1 THD - Technische Hochschule Deggendorf, University of Applied Sciences, D-94469 Deggendorf, rolf.rascher@th- \\ deg.de \\ 2 FISBA AG, Rorschacherstrasse 268, CH-9016 Switzerland, oliver.faehnle@fisba.com \\ 3 University of Arizona, Tucson, USA, letter2dwk@hotmail.com
}

\begin{abstract}
A novel fabrication parameter controlling method for laser polishing processes called CLasso (Control of LASer Surface Optimization) is presented, monitoring within the footprint the smoothening process as well as the removal of ssd in situ. Therefore, it is possible to determine and control the optimum dwell time a footprint needs to stay at a certain point before moving further enabling a more stable and cost optimized polishing.
\end{abstract}

\section{Introduction}

Among the various sub-aperture polishing techniques of glass existing, laser polishing [1] distinguishes itself by its scalable non-contact polishing spot as well as by the absence of any solid contact between tool and workpiece. Polishing is achieved by local absorption of laser power within the polishing spot (the footprint) causing the surface and its subsurface region to melt and flow reducing surface roughness and removing subsurface damage (ssd). To that aim, setting up process parameters is a tradeoff between continuous wave at lower laser power values melting the surface and near surface regions gently but risking the generation of shape deviations and mid-spatial frequencies due to material flow; and on the other hand the application of a pulsed laser illumination at high power values avoiding changes in shape and mid-spatials but risking high values of surface and subsurface tension caused by the high temperature gradients generated which might even cause local cracking. In both cases, the risk of vaporization of material is existing which might cause redepositioning of material by the laser beam being scanned along the surface.

In the following, a new fabrication parameter controlling (FPC) method for laser polishing processes called C-Lasso (Control of LASer Surface Optimization, Patent Pending) is being presented, enabling the determination and control of optimum local dwell times and process parameters.

\section{C-Lasso: dwell time monitoring}

It has been demonstrated that monitoring the intensity of a laser beam that is being reflected at an optical surface from within the sample while its outside is being abrasively ground and polished, surface roughness level reductions can be monitored in process on machine [2]. Consequently, applying intensity-detecting Internal Reflective Microscopy, iIRM [3], polishing time and cost can be optimized. Fig. 1 shows the usually applied setting up onto a standard overarm polisher where the signal is being detected touching the surface under test optically from within the sample while being polished from the outside. Fig. 2 demonstrateds the resolution of the IRM measuring technique: two samples have been polished and measured by whitelight interferometry previously. Subsequently, both have been measured ny IRM demonstrating its capability to destinguish levels of surface roughness in the sub nanometer range. Fig. 3 shows the IRM resolution for the detection of sleeves and scratched caused during polishing. 

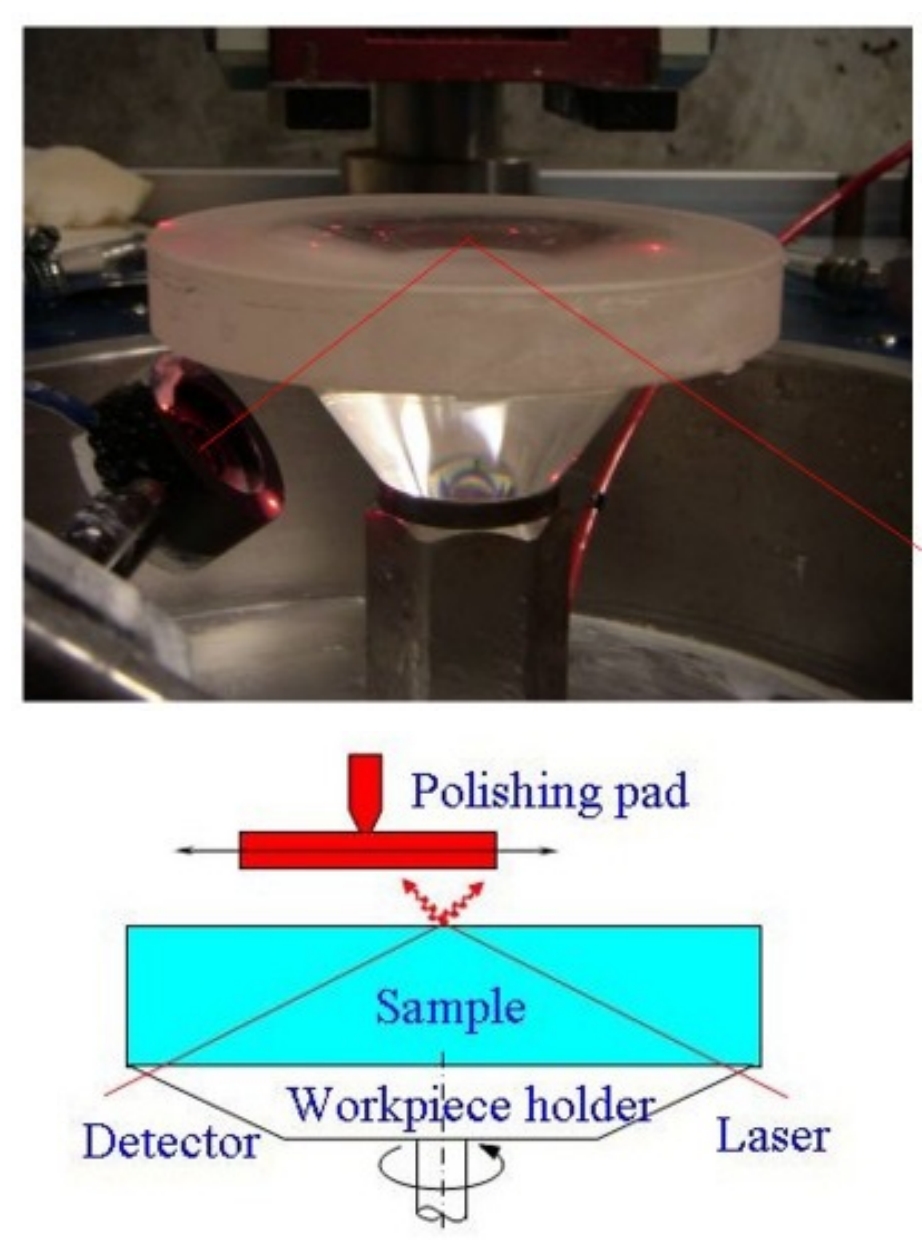

Fig.1 ilRM: while the sample is being polished, the intensity of a laser beam is being detected that is reflected from within the material at the surface under test.

Besides that, the influence of changing process parameters (such as e.g. the $\mathrm{pH}$ values of polishing slurries) to the generated surface roughness level being generated can be observed in situ, enabling the determination of the optimum set of polishing process parameters [4].

ilRM method has been developed further (see Fig.4 and [5]) to enable monitoring of laser polishing processes of fused silica. A HeNe laser beam is being reflected from within the sample at the surface under test and the intensity of its reflected beam is being monitured. The internal local sub-aperture surface area under test is chosen to be located at the very spot where the laser polishing footprint is being positioned from the outside of the sample, melting its surface locally. 


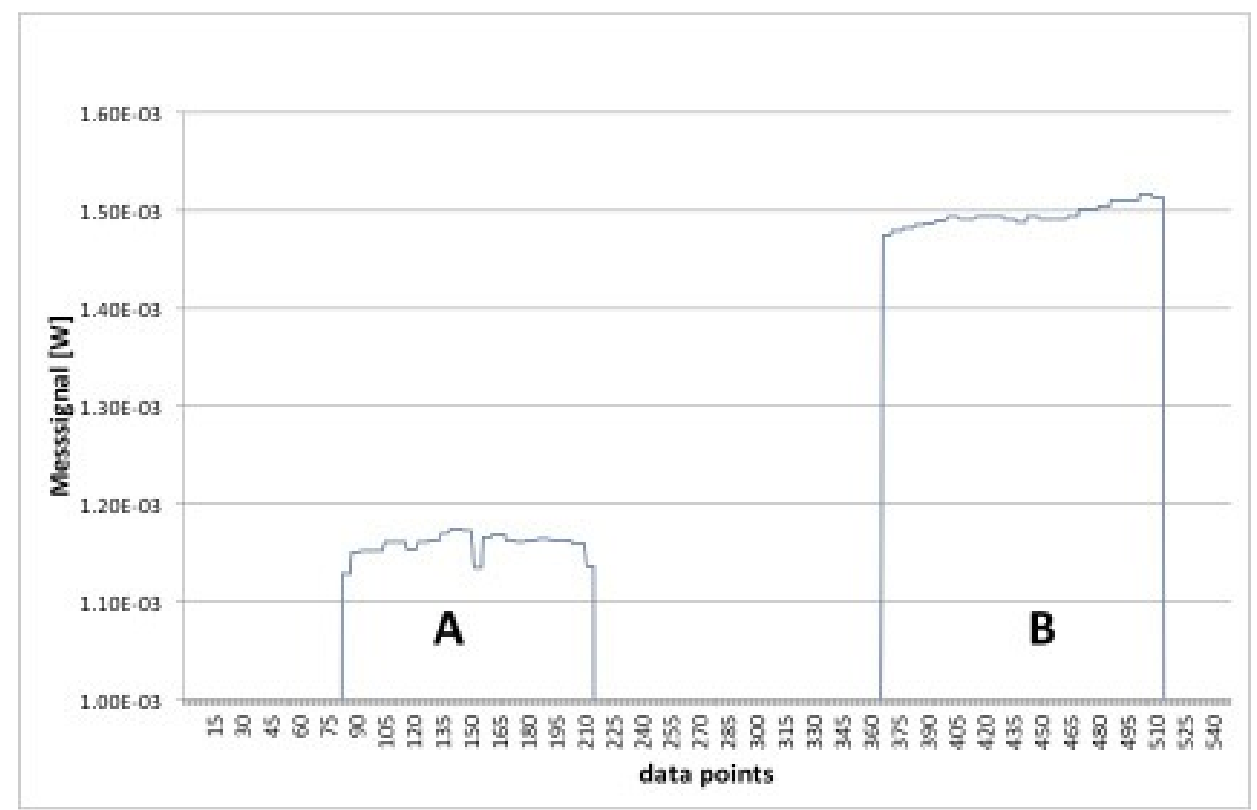

Fig.2 ilRM measurements of sample $A(S q=1.1 \mathrm{~nm} \mathrm{rms})$ and sample $B(S q=0.6 \mathrm{~nm}$ rms)

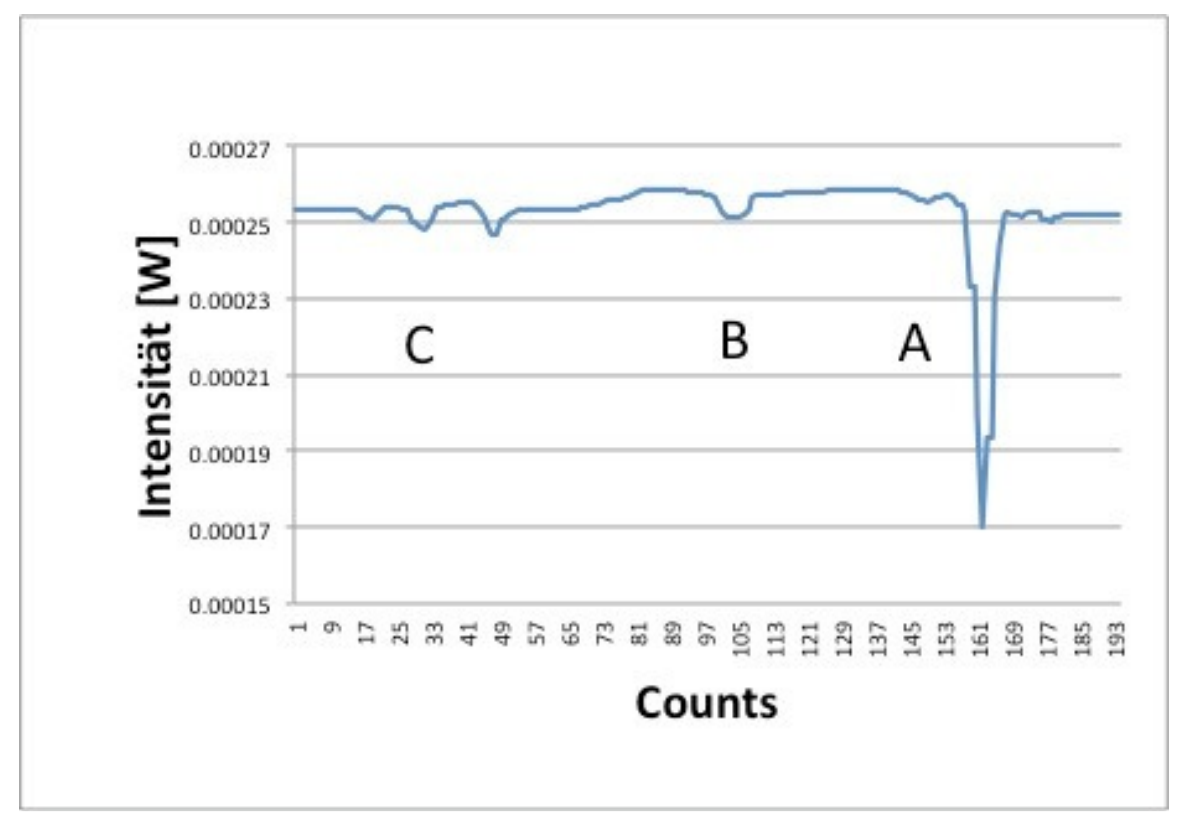

Fig.3 ilRM measurement of the generated grooves $A$ (900 $\mathrm{nm}$ deep brittle scratch), $B$ (150 nm deep ductile sleeve) and C (several polishing sleeves: the smaller ones of which are about $100 \mathrm{~nm}$ deep). 
Thanks to the fact, that fused silica is not transparent at the operating laser wavelenth of the applied $\mathrm{CO} 2$ laser, the intensity of the reflected beam can be monitored enabling a in situ control of the laser polishing process. C-Lasso (Control of LASer Surface Optimization, Patent Pending) monitors within the laser footprint the surface smoothening process as well as the removal of ssd caused by the chosen set of laser polishing parameters being applied (such as e.g. dwell time and applied level of laser power).

(a)

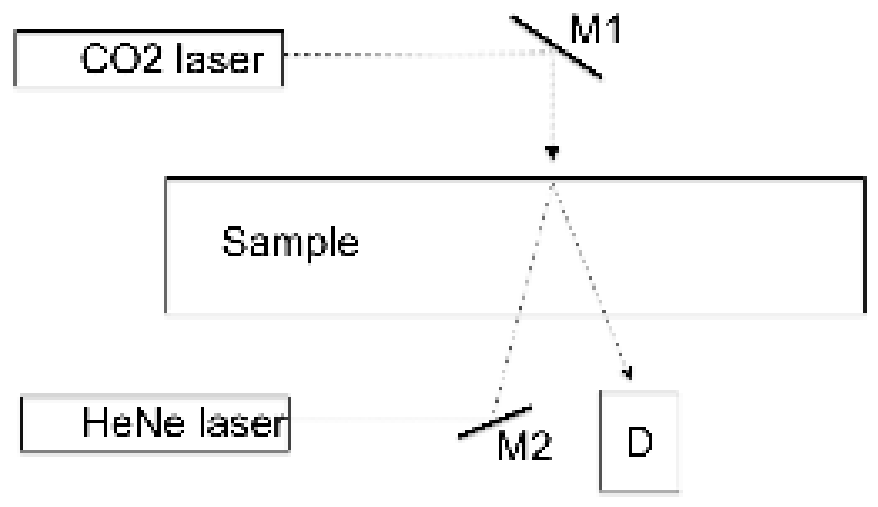

(b)

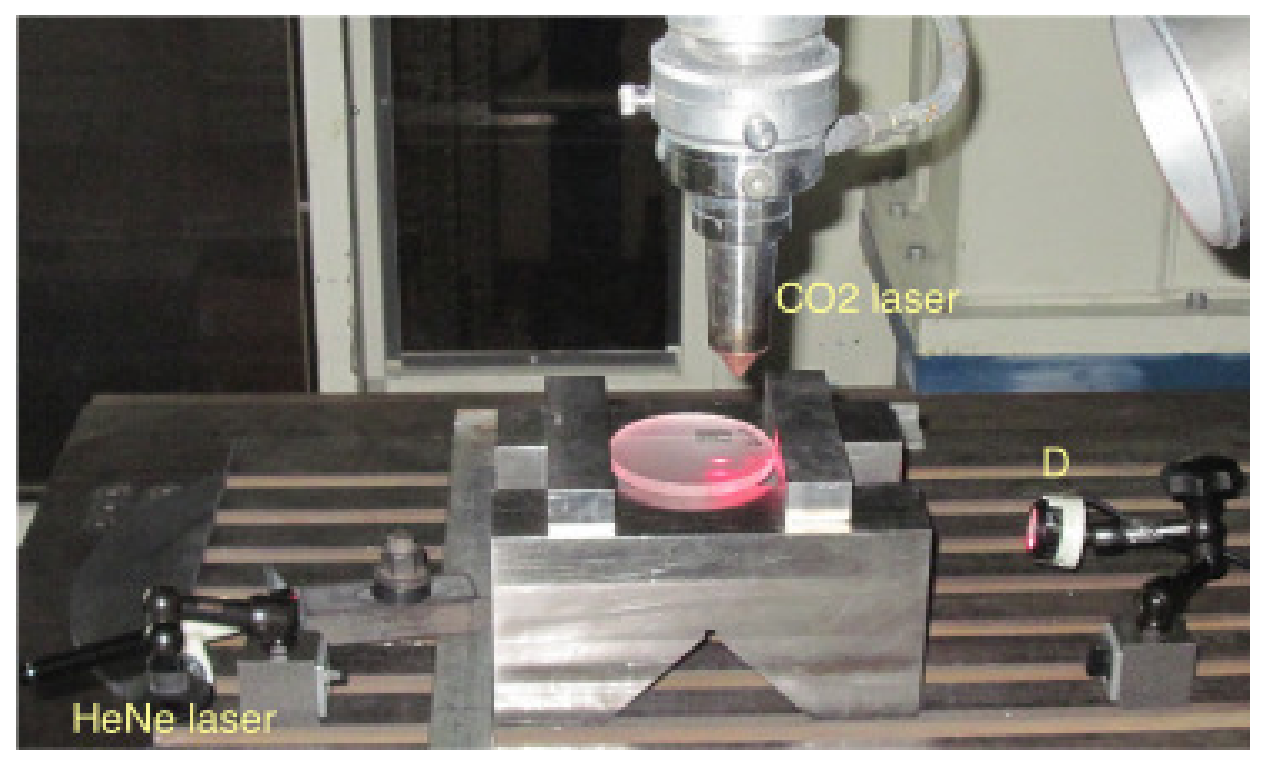

Fig.4 (a) Sketch of the C-Lasso set up for in situ monitoring of $\mathrm{CO} 2$ laser polishing process. While the sample is locally being laser polished (see scanning mirror M1), the level of surface roughness within the laser footprint is being monitored by detecting the intensity of a HeNe laser beam that is being reflected at the $\mathrm{CO} 2$ footprint area from within the sample. (b) Set-up used for the presented proof-of-principle generating C-Lasso's "First light". 
Fig. 5 shows a typical C-Lasso signal detected by monitoring surface roughness reduction during the laser polishing process. A previously fine ground fused silica surface (see Fig.6) is locally being laser polished while the footpront area is being monitored by C-Lasso.

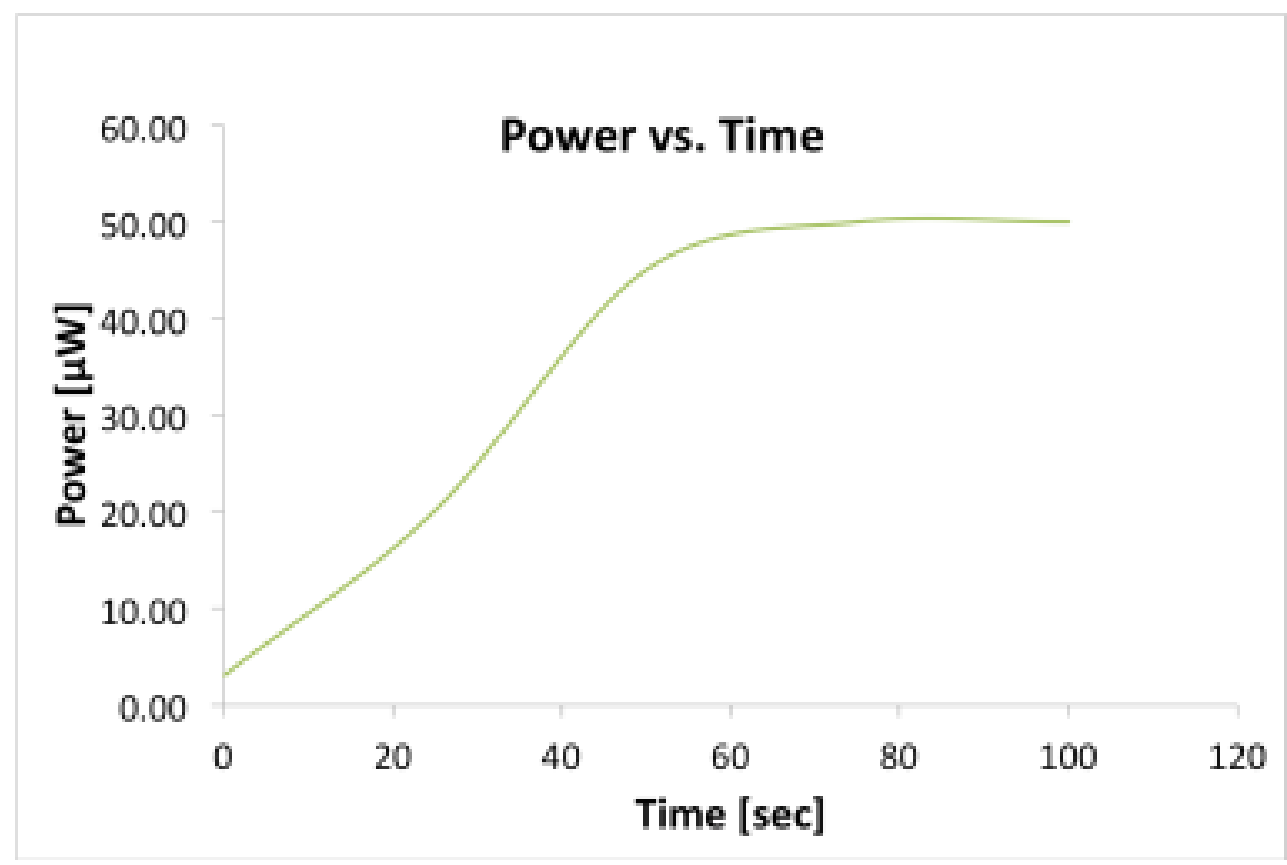

Fig.5 C-Lasso signal detected in situ while the footprint is locally laser polishing a fused silica surface: starting from a ground surface with about $300 \mathrm{~nm}$ rms a shiny surface is being generated featuring less than $5 \mathrm{~nm} r \mathrm{rs}$.

As the level of surface roughness decreases due to the melting process, the intensity of the reflected C-Lasso beam increases until the final surface quality has been reached and the CLasso signal reaches a plateau. From that point on, any further laser polishing does not increase surface quality anymore.

\section{Conclusions}

It has been demonstrated that by applying C-Lasso method (Patent Pending), it is possible to determine and control the minimum dwell time a laser polishing footprint needs to stay at a certain point before moving further. Consequently, C-Lasso enables the optimization of laser polishing by avoiding unneccessarily long local dwell times minimizing the risk of generating shape deviations, ssd and mid-spatials as well as the vaporization of glass. Besides that, after having reached the eventuel C-Lasso plateau, the influence of process parameters, such e.g. the applied $\mathrm{CO} 2$ laser power, to the generated level of surface roughness can me monitored in situ enabling the detemination of an optimized set of laser polishing parameters. Currently, C-Lasso is being implemented into a laser polishing set-up to be employed for further investigations of laser polishing parameters in situ. 
a)

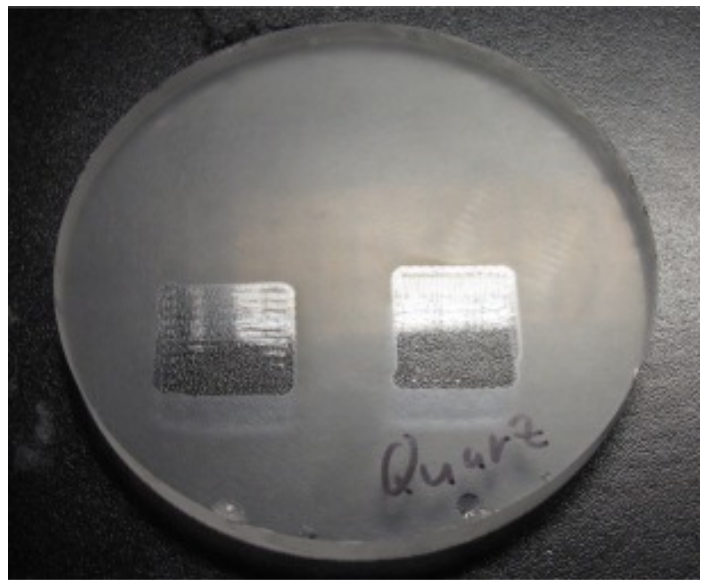

b)

c)

\begin{tabular}{|cc|}
\hline Results & \\
\hline Sa 1.964 & $\mathrm{~nm}$ \\
Sq 2.587 & $\mathrm{~nm}$ \\
Sz 36.329 & $\mathrm{~nm}$ \\
\hline Size X 383.733 & $\mu \mathrm{m}$ \\
Size Y 383.733 & $\mu \mathrm{m}$ \\
FOV $0.42 \times 0.42 \mathrm{~mm}$ \\
\hline
\end{tabular}

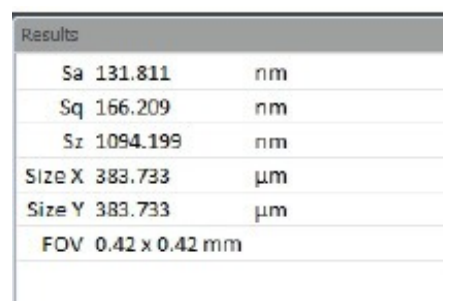

Fig.6 Starting and final roughness of a typical laser polished fused sample (the in situ in process monitoring of which is shown in Fig.2) measured by white light interferometry to be $160 \mathrm{~nm} \mathrm{rms}$ before processing and about $2 \mathrm{~nm} \mathrm{rms}$ of the finished part.

\section{REFERENCES}

[1] A. Richmann, E. Willenborg, and K. Wissenbach, "Laser Polishing of Fused Silica," in International Optical Design Conference and Optical Fabrication and Testing , OSA Technical Digest (CD) (Optical Society of America, 2010), paper OTuC2.

[2] O. Faehnle, "Process optimization in optical fabrication", SPIE Journal on Optical Engineering 0001;55(3):035106. doi:10.1117/1.OE.55.3.035106., 2016

[3] O. Faehnle, F. Zygalsky, E. Langenbach, F. Weimer, M. Kahl and A. Ettemeyer, "In situ monitoring of surface roughness and contamination of polishing processes", EOSMTOC conference on "Manufacturing and Testing of Optical Components ", European Optical Society (EOS), München, Germany, June 2015

[4] O.Fähnle, "In process monitoring of optics fabrication", SPIE conference on "Precision Optics Manufacturing", Deggendorf Institute of Technology, Deggendorf, Germany, April 2017

[5] O. Faehnle, R. Rascher, C. Vogt, and D. W. Kim, "Closed-loop laser polishing using inprocess surface finish metrology," Appl. Opt. 57 (2018). (in press) 